\title{
Briefly Legal: Need for Glucose Screening After Fetal Distress, A Misadventure in the Normal Nursery
}

Maureen E. Sims, M.D., Barry Schifrin, M.D,

Facts of the Case:

A 27 y/o primigravida patient presented at 40 2/7 weeks gestation with vaginal spotting, back pain, and contractions. To that point, her pregnancy had been uncomplicated, although her Group B Streptococcus status was unknown. External fetal monitoring, initiated on admission, revealed a Category 1 tracing, which evolved in association with misoprostol, oxytocin, excessive uterine activity, and epidural anesthesia to a Category 2 for the remaining 8 hours of labor. During the last 2 hours, repetitive late decelerations and minimal variability were apparent (a Category III tracing). The mother was given oxygen intermittently, placed in the left lateral position for pushing, and given multiple fluid boluses containing glucose. Because of deterioration of the fetal heart rate pattern, an emergency cesarean section was performed with a vacuum assist of the fetal head. At delivery, a nuchal cord was easily reduced. Scalp swelling and bruising in the midspinal area were noted. At birth, the baby was appropriate for gestational age (AGA), with a birth weight of $3260-\mathrm{g}$, length $50.8 \mathrm{~cm}$, and head circumference of $36 \mathrm{~cm}$. Apgar scores were $6^{1}$ and $8^{5}$. The cord was clamped after 30 seconds. Umbilical cord blood gas analysis revealed: Artery pH 7.09, pC02 74mm Hg, p02 10 mm Hg, Base excess -8.8; Vein: pH of 7.2, pC02 $56 \mathrm{mmHg}$, p02 $28 \mathrm{mmHg}$ base excess of 7.2 .

\section{"External fetal monitoring, initiated on admission, revealed a Category 1 tracing, which evolved in association with misoprostol, oxytocin, excessive uterine activity, and epidural anesthesia to a Category 2 for the remaining 8 hours of labor. During the last 2 hours, repetitive late decelerations and minimal variability were apparent (a Category III tracing). "}

The baby received blow-by oxygen from a $1^{\text {st }}$-year pediatric resident and a labor and delivery nurse - neither an RT nor a senior neonatal attending was present despite that indication for the emergency cesarean section was fetal distress. At 20 minutes, it was noted that he had a normal physical examination except for elevation of the heart rate (HR) 186 beats per minute (bpm), a respiratory rate of 64 breaths per min (BPM), intercostal retractions with a "rare cry."

The plaintiff obstetrical experts were critical of the defendant obstetrician and nurses for failing to appreciate that this fetus was not tolerating the labor well. The prospects of safe vaginal delivery were significantly diminished despite the excessive uterine activity. They averred that the delay in performing a cesarean section earlier subjected the fetus to a prolonged period of hypoxia and ischemia. The obstetrical expert for the defendant disagreed. The mother stated in her deposition that she was not asked permission for a vacuum assist. Depositions of the treating nurses and physicians for the baby revealed that they were unaware of a cord gas being sent and did not know the significance of arterial cord acidemia. The plaintiff experts were critical of this lack of awareness as well as the cord arterial acidosis on the newly born baby and the need for monitoring for a baby born after this stress. The plaintiff experts were critical of the hospital for not having the attending pediatrician or neonatologist informed of this baby's birth. These findings mandated an evaluation from an experienced provider.

The infant and was ultimately sent to the normal newborn nursery. The pediatric resident wrote routine orders, which included rooming-in with the mother and the option for breastfeeding. The hospital protocol did not require the pediatric resident to notify the on-call attending pediatrician. An hour after birth, the nurse called the on-call pediatrician to inform him of the birth of a new baby who was not interested in breastfeeding and who remained symptomatic.

In their depositions, the pediatric nurse stated that she did not inform the pediatrician on-call about the emergency cesarean section for fetal distress or the cord blood gases and the pediatrician admitted that he did not inquire about such details. He said that he assumed it was a normal baby who was transitioning. The plaintiff neonatologist pointed out that not only was the history important, but the baby's symptomatology required attention and investigation. The attending pediatrician on-call ordered the baby to be transferred to the normal nursery from the rooming-in with the mother to where he could be "closely observed." The baby arrived in the normal nursery about 1.5 hours after birth and was cared for by a labor and delivery nurse. In addition to the persisted high HR and RR and intercostal retractions, the nurse noted the development of lethargy.

"In their depositions, the pediatric
nurse stated that she did not inform
the pediatrician on-call about the
emergency cesarean section for fetal
distress or the cord blood gases and
the pediatrician admitted that he did not
inquire about such details. He said that he
assumed it was a normal baby who was
transitioning."

The plaintiff's expert neonatologist was critical of the nurse and the on-call pediatrician allowing this symptomatic baby to be cared for in the normal nursery rather than in the NICU, where dedicated surveillance was available. The plaintiff's experts drew attention to the differential diagnosis, which included hypoxic-ischemic injury, intracranial hemorrhage, as well as hypoglycemia. Given the history of prolonged fetal distress and difficult delivery, hypoglycemia should be ruled 
out irrespective of the remainder of the differential diagnosis. Any symptomatic baby without known reasons for the symptoms mandated the determination of a serum glucose level.

The defense experts countered that since the baby was at term and appropriate for gestational age (AGA) and the mother was not known to be diabetic, the (prenatal historical) risk factors did not put this baby at risk for hypoglycemia. Plaintiff experts strongly disagreed. The symptoms immediately after birth were probably secondary to the response from the ongoing hypoxic-ischemic insults and the mechanical forces exerted on the fetal head, given the obstetrician's difficulty in extracting the fetal head at birth. There was no evaluation of blood gases (despite the indication of fetal distress for operative delivery). Nor was a chest radiograph obtained. It was not possible to be more specific except to opine he was acidotic at the time of birth and had increased metabolic demands with his increased work of breathing. While the immediate glucose levels in the neonate are close to those of the mother's glucose concentration at birth, perinatal stress mobilizes glucose via the release of catecholamines. When the maternal supply is cut off at birth, neonatal hypoglycemia becomes an etiology that should have been considered, as well as several other etiologies of the baby's symptoms. A bedside glucose evaluation is simple to obtain and rapidly available: if found to be abnormally low, it allows easy intervention. But if low serum glucose remains, it risks an adverse outcome, including seizures and neurological injury. Intrapartum hypoxia and ischemia coupled with hypoglycemia are far worse together compared to either alone.

\section{"The plaintiff experts explained that it was below the standard of care to fail to evaluate this baby with increasing symptoms properly. The nurses should have insisted that the physician come to the bedside, and protocols should have been in place for a bedside glucose evaluation."}

The baby began having intermittent desaturations, which responded to blow-by oxygen. The nurse called the on-call pediatrician again to inform him of the desaturations. The plaintiff experts explained that it was below the standard of care to fail to evaluate this baby with increasing symptoms properly. The nurses should have insisted that the physician come to the bedside, and protocols should have been in place for a bedside glucose evaluation. Plaintiff experts pointed out that the baby had experienced intrapartum distress, likely had an abnormal arterial cord gas and was symptomatic. He should have been admitted to the Newborn Intensive Care Unit (NICU). The plaintiff experts were critical of the nurses for not advocating for this baby. They should have known that a baby born with non-reassuring tracings, a vacuum assist, cord acidemia required higher-level care than that provided in a normal nursery. The symptomatology by itself was sufficient to mandate NICU admission. They should have insisted upon a physician's presence, gone through their chain of command if they could not persuade the physician to come to the bedside and admit the baby to the NICU or directly called the neonatologist on-call.
Because the oxygen desaturations continued, the nurse, at 1.5 hours of life, did a point of care glucose assessment and found it below the readable range $(<10 \mathrm{mg} / \mathrm{dL})$. The nurse informed the on-call pediatrician about the low glucose and received an order to gavage fed the baby $25 \mathrm{ml}$ of formula and to recheck the glucose after 20 minutes. The plaintiff pointed out that it was inappropriate to gavage feed a baby with a prohibitively low serum glucose level. When a baby who should be nippling is too sick to do so, gavage feeding is not an appropriate substitute and is potentially dangerous. The likely reason this baby did not feed was the several hours of hypoxemia and acidosis in utero. Furthermore, hypoglycemic babies tend not to want to feed. Rather, the standard of care required the immediate administration of an intravenous bolus of glucose. A point of care glucose was repeated after the gavage feed; it was again below the readable range. The infant's $H R$ and $R R$ remained high, the oxygen saturations were intermittently low, and the infant remained lethargic. Several conversations between the nurse and the physician occurred while the baby was in the normal nursery, with the physician eventually assuring the nurse he would come in soon.

\section{"Two and a half hours after birth, the pediatrician arrived at the bedside. Two additional bedside glucose evaluations were again unreadable. The pediatrician called the neonatologist requesting admission to the NICU."}

Two and a half hours after birth, the pediatrician arrived at the bedside. Two additional bedside glucose evaluations were again unreadable. The pediatrician called the neonatologist requesting admission to the NICU. The pediatrician entered several orders at this point: $15 \mathrm{ml}$ formula to be gavaged, a bolus of $6 \mathrm{ml}$ of $10 \%$ dextrose, a complete blood count (CBC) and blood culture, a peripheral intravenous line, ampicillin and gentamicin, and admission to the NICU. At this point, the baby had decreased tone and was minimally responsive; his $\mathrm{HR}$ was $148 \mathrm{bpm}$, temperature $36.5^{\circ} \mathrm{C}$, a RR of $66 \mathrm{BPM}$, a blood pressure of $60 / 38$ with a mean of $45 \mathrm{~mm} \mathrm{Hg}$. At $3 \frac{1}{2}$ hours after birth and 2 hours after the initial unreadable glucose assessment, a bolus of $6 \mathrm{ml}$ of $10 \%$ dextrose was given intravenously.

\section{"The baby was discharged at two weeks on no medications and breastfeeding. The child had severe developmental delay, cerebral palsy, and a seizure disorder at a three-year follow-up examination."}

During the first 24 hour period in the NICU, the baby remained minimally responsive. His glucose values ranged from $27-47 \mathrm{mg} /$ $\mathrm{dL}$. A lumbar puncture revealed no evidence of infection. During the following 24 hours, with higher, more stable glucose values, seizures began for which phenobarbital was provided. Disseminated intravascular coagulopathy (DIC) developed with elevated liver enzymes. A cranial ultrasound showed a region suspicious 
for parenchymal hemorrhage in the temporal, occipital region. An MRI showed intraventricular hemorrhage, scattered bilateral parenchymal hemorrhages, and left cerebellar and posterior fossa subdural hemorrhages. The baby was discharged at two weeks on no medications and breastfeeding. The child had severe developmental delay, cerebral palsy, and a seizure disorder at a threeyear follow-up examination.

The obstetrical staff was sued for:

1. Failure to properly evaluate and timely respond to the abnormal FHR tracing.

2. Failure to appreciate the diminished feasibility of safe vaginal delivery

3. Failure to timely deliver by cesarean section.

The hospital was sued for:

1. Allowing this symptomatic baby to remain with the mother for an hour, then in a normal nursery,

2. Delayed assessment of glucose

3. Failing to notify the attending pediatrician promptly

4. Failure to appropriately supervise the pediatric resident

5. Not appreciating the emergency nature of profound hypoglycemia

6. Not intervening timely with intravenous glucose

7. Not advocating for this baby by insisting on the presence and appropriate actions from the on-call pediatrician.

8. Failure to go up the chain of command.

\section{"The on-call attending pediatrician was sued for not responding and intervening appropriately to the baby timely. Each party blamed the others for the adverse outcome. All parties settled."}

The on-call attending pediatrician was sued for not responding and intervening appropriately to the baby timely. Each party blamed the others for the adverse outcome. All parties settled.

\section{Discussion}

\section{General:}

Glucose is the major energy source for fetuses and neonates. Fetal fuel metabolism is based primarily on glucose oxidation, which is supplied from maternal plasma glucose whose levels are regulated by maternal insulin secretion that does not cross the placenta. Fetal insulin secretion increases cellular glucose uptake, deposits glucose as glycogen, facilitates lipogenesis, and inhibits the breakdown of fatty acids triglycerides in preparation for the energy demands of the newborn. Thus, while fetal insulin is responsive to plasma glucose concentrations, its response in the normal newborn is delayed compared to the adult or the infant of the diabetic mother. Its primary function is the regulation of fetal growth.

Within 1 to 1.5 hours after birth, blood glucose concentrations have fallen rapidly by $20-25 \mathrm{mg} / \mathrm{dL}$ from their fetal glucose concentrations to a nadir of about $55-60 \mathrm{mg} / \mathrm{dL}$. By 3 hours, they have risen and stabilized even in the absence of any intake. During this time, plasma insulin levels fall, and plasma glucagon levels rise. They are presumably mediated through a surge in catecholamines and other counterregulatory hormones. By 12-24 hours, blood glucose concentrations stabilize between 43 and $90 \mathrm{mg} / \mathrm{dL}$ reaching a steady-state by 2 to 4 days of life. These relationships are altered under various conditions, including maternal diabetes, $\mathrm{Rh}$ isoimmunization, and preeclampsia. Further, during severe fetal distress, where the supply of glucose from the mother is curtailed by placental insufficiency, cord compression, or cerebral ischemia, the fetus obtains glucose from stored glycogen, and fetal insulin is required to move glucose into the cells for energy.

Although all organs can use glucose, the human brain uses it almost exclusively as a substrate for energy metabolism. The high brain-to-bodyweight ratio in the newborn results in a proportionately higher demand for glucose compared with the capacity for glucose production than that encountered later in life, with cerebral glucose use accounting for as much as $90 \%$ of total glucose consumption. The fetus is exposed to circulating glucose concentrations about 9 $\mathrm{mg} / \mathrm{dL}$ below those of maternal plasma, which normally are 70-90 $\mathrm{mg} / \mathrm{dL}$. With the abrupt severing of the umbilical cord, exogenous glucose supply is terminated, and nutrient and neonatal glucose levels fall. The newly born embarks upon a transition of complex and physiologic challenges that require metabolic adaptation to maintain appropriate plasma glucose concentrations.

First, a word must be said about the normal response to a decreasing glucose concentration in older babies, children, and adults. In normal individuals, the maintenance of normal plasma glucose concentrations is highly protected. The two metabolic responses to hypoglycemia are 1) to lower insulin levels and 2) to mobilize alternate fuel sources. The metabolic response that opposes or counters the insulin secretion to maintain euglycemia is referred to as a "counterregulatory response." The main counterregulatory hormones are glucagon, epinephrine, cortisol, and growth hormone. The other response to maintain euglycemia is to tap into alternate fuel sources, namely lactate, a major gluconeogenic substrate, $\beta$-hydroxybutyrate $(\mathrm{BHB})$, a major ketone, and free fatty acids, a robust energy source released from adipose tissue.

These mechanisms to regulate glucose are sluggish at birth, putting the newly born at risk for hypoglycemia. These mechanisms are especially challenged in babies born with increased glucose demands or when exogenous or endogenous glucose supply is limited. Immediately after birth, the newborn experiences a drop in glucose. In the healthy full-term infant, the nadir usually is no lower than $40 \mathrm{mg} / \mathrm{dL}$ between 30 and 90 minutes after birth. Thereafter, the plasma glucose concentration rises and is maintained at a steady level of about $55-60 \mathrm{mg} / \mathrm{dL}$ by $1-2$ hours. It steadily rises over the next 1-2 days and is in the normal range for infants, 
children, and adults. The plasma glucose concentration during the first $24 \mathrm{~h}$ after birth in healthy, asymptomatic breastfed babies has been reported to have a mean of $65 \mathrm{mg} / \mathrm{dL}$ compared to formulafed babies with an average of $72 \mathrm{mg} / \mathrm{dL}$.

\section{Clinical Categorization}

A reasonable classification of neonatal hypoglycemia is to categorize them into four groups.:

1. "Transitional neonatal hypoglycemia" or "early transitional-adaptive hypoglycemia" is a common variety of neonatal hypoglycemia and occurs in the first 3-12 hours after birth. The pathophysiology involves the failure of one or more of the early adaptive responses to birth. This "normal hypoglycemia" is most likely due to the persistence of the fetal pattern of insulin regulation resulting in higher insulin levels for the newly born. Speculation is that the low glucose threshold for suppressing insulin release at birth reflects a fetal islet adaptation that allows the fetus to secrete sufficient insulin to maintain fetal growth even at fetal glucose concentrations that are lower than in the mother and also at times when maternal glucose concentrations are reduced (e.g., during fasting or limited calorie consumption). An interesting finding of neonatal hypoglycemia in normal newborns is that the plasma glucose levels are lowest early on the first day of life and then progressively increase. Also of interest is that during transitional neonatal hypoglycemia in normal infants, plasma glucose concentrations are remarkably stable and relatively unaffected by the timing of initial feeding or interval between feedings. This data should be considered when relying on preprandial glucose assessments.

2. Impaired metabolic adaptation is a heterogeneous group and relatively common in neonates. These babies are born with various conditions that make them at risk for hypoglycemia. Although there is overlap concerning the pathophysiology of hypoglycemia in the various conditions, a predominant feature is a lower glucose threshold for suppression of insulin secretion than expected and results in impaired glucose and ketone production. Included in this group are babies born with various conditions, including asphyxia, preterm, SGA, IDM, congenital heart disease, infection, and some conditions that develop after birth, such as iatrogenic cold stress

3. Intrauterine growth restriction (IUGR), where undernutrition is the dominant feature. This disturbance affects glycogen and lipid stores, gluconeogenic capacity, and ketone production.

4. Severe recurrent hypoglycemia is secondary to specific primary enzymatic or metabolic-endocrine abnormalities involving glucose homeostasis.

\section{"It can be challenging to identify and distinguish newborn infants having transitional hypoglycemia from several other conditions during the first 48 hours."}

It can be challenging to identify and distinguish newborn infants having transitional hypoglycemia from several other conditions during the first 48 hours.

\section{Alternate Fuels:}

While the newborn brain can use alternative metabolic substrates, such as lactate and ketone bodies, the supply of these is limited, and the newborn's immature counterregulatory response limits their availability. Lactate provides a potential alternative fuel in the first $48 \mathrm{~h}$ and ketones may be available on days 3-4, but each can provide only a small proportion of total brain energy requirements. Neither lactate nor BHB is likely to provide neuroprotection during early or acute hypoglycemia. Hypoglycemia, irrespective of feeding or fasting, suppresses plasma-free fatty acids (FFA) as well as ketones. It cannot be assumed that ketones are available as an alternative fuel to support brain metabolism when normal neonates develop hypoglycemia or that breastfed babies are protected against potential adverse effects of hypoglycemia by ketones if their postnatal fasting period extends too long. Interestingly, pregnant women develop hyperketonemia much earlier than non-pregnant women (accelerated starvation), thereby providing the fetus with alternative fuel to protect the brain when plasma glucose is low, thus making it unnecessary for the fetus to switch off insulin secretion and thereby avoid limiting growth and development.

\section{Defining Neonatal Hypoglycemia:}

The definition of a blood glucose level below which hypoglycemia should be designated is complex and cannot be based on a single number uniformly applied to all infants. Attempts at such definitions have been based on statistical thresholds derived from the study of serial changes of blood glucose in normal infants; on operational thresholds based on blood glucose levels and the presence or absence of symptoms or risk factors; on neurophysiologi$\mathrm{cal}$ thresholds based on changes in brain stem auditory evoked responses (BSAERs), cerebral blood flow (CBF), or cerebral glucose metabolism; and on neurological outcome thresholds based on neurodevelopmental outcome as a function of different blood glucose levels. What is needed is the identification of the threshold at which treatment would prevent brain injury.

\section{"However, thresholds vary depending on gestational age, postnatal age, concurrent metabolic demands, co-morbidities, intrapartum challenges, and availability of alternative metabolic fuels. Further, even if healthy term babies have low glucose concentrations during the transition, their reference ranges should not be normative for infants who have impaired metabolic adaptation."}

However, thresholds vary depending on gestational age, postnatal age, concurrent metabolic demands, co-morbidities, intrapartum challenges, and availability of alternative metabolic fuels. Further, even if healthy term babies have low glucose concentrations during the transition, their reference ranges should not be normative for infants who have impaired metabolic adaptation.

For example, a term baby with a benign prenatal period who then is subjected to hypoxic and ischemic challenges during labor and delivery is metabolically very different from the healthy term baby with a normal intrapartum course. Infants who are ill enough to be admitted to the NICU automatically will receive bedside glucose evaluations soon after admission and receive the needed inter- 
ventions. However, the fetus who had a challenging intrapartum course who is compromised but has a heart rate and is breathing (even if he needed positive pressure briefly to achieve a normal respiratory drive), who has decreased tone, weak or absent cry, whose color is "off" often will be roomed-in with mother or be admitted to the normal nursery to be "closely observed." Glucose evaluations are only performed when symptoms are appreciated.

\section{Screening for Hypoglycemia:}

Routine screening in an acute setting occurs on babies admitted from the delivery suite to the NICU, with the usual reason for admission being immaturity, small size, cardiovascular or respiratory instability, or significant anomalies. It is common practice in developed countries to screen certain categories of asymptomatic babies known to be at risk for hypoglycemia.

Routine screening for asymptomatic babies in a non-acute setting occurs for infants of diabetic mothers (IDM), and small for gestational age (SGA), large for gestational age (LGA), and the late preterm (LPT, 34 - 36 6/7 weeks gestation) gestations. Such babies are well known to be at risk, and even when appearing healthy and stable, they room in with their mother or are cared for in the normal nursery. The initial screening is usually done $1-2 \mathrm{~h}$ after birth, followed by regular measurements over the next 12-48 $\mathrm{h}$ until the baby is consistently euglycemic.

\section{Escaping the Safety Net:}

Routine screening is not done on a large swath of asymptomatic babies who nevertheless are at risk for hypoglycemia while rooming in with their mother or residing in the normal nursery. These are the babies who appear healthy and stable immediately after birth because neonatal adaptation appears satisfactory. But in fact, they have a history of prenatal or perinatal stressors that put them at risk for hypoglycemia. At times, stability is achieved in the delivery room after the baby is born because they briefly receive positive pressure ventilation or blow-by oxygen. Sometimes the tone is decreased, or the cry is not present or strong. Sometimes the color is not normal. They enter areas where they are presumed to be normal or close enough and will be not be "observed closely." Many among them are born a) after fetal distress, difficult deliveries, cesarean section for intolerance to labor or difficult deliveries as in the case presented b) to mothers with hypertension; $c$ ) to mothers who received glucose infusions, especially boluses during fetal decompensation intervals; d) with IUGR e). with post dates f) with higher length compared to weight (low PI), g) born to mothers who received sympathomimetics (terbutaline, ritodrine,) and propranolol and finally, babies affected by Rh sensitization.

\section{"Concern for substantial neurologic morbidity in neonates with hypoglycemia has led to the promulgation of guidelines by the American Academy of Pediatrics $(A A P)$ in 2011, the Pediatric Endocrine Society (PES) in 2015, and most recently by Dr. Volpe (2019)."}

\section{Treatment and Justification:}

Concern for substantial neurologic morbidity in neonates with hypoglycemia has led to the promulgation of guidelines by the
American Academy of Pediatrics (AAP) in 2011, the Pediatric Endocrine Society (PES) in 2015, and most recently by Dr. Volpe (2019). In reviewing the literature and guidelines, one must be careful to note whether the values represent mean glucose values or a threshold range for glucose values (e.g., below the $5^{\text {th }}$ percentile (AAP). Dr. Volpe favors the following approach: infants at risk for impaired metabolic adaptation (babies who experienced in utero hypoxic-ischemic insult, IDM, and SGA) even if asymptomatic with a single low blood level to be treated with intravenous glucose. Volpe justifies this approach based on:

1. Neurophysiological, epidemiological, and clinical observations that levels less than $50 \mathrm{mg} / \mathrm{dL}$ can be associated with evidence for neurophysiological or neurodevelopmental dysfunction;

2. The PET observation that cerebral glucose utilization in the premature infant may be limited by glucose transport at levels of plasma glucose less than approximately $54 \mathrm{mg} / \mathrm{dL}$;

3. The likelihood that degrees of hypoglycemia not sufficient to cause brain injury alone may do so when combined with other factors deleterious to the central nervous system;

4. The lack of precise information regarding the level of blood glucose below which neuronal injury is likely to occur.

5. The realization that the parietal-occipital region and higher visual functions are most sensitive to and that such cortical functions have not been carefully studied in most follow-up reports; and

6. The ample experimental evidence that blood glucose levels are not accurate predictors of brain glucose levels, particularly in states such as asphyxia or seizures.

The physician must consider the status of both hypoglycemia and cerebral glucose delivery (i.e., CBF) and blood glucose content and cerebral glucose utilization. Once a decision is made to treat an infant for hypoglycemia, a bolus of $2 \mathrm{~mL} / \mathrm{kg} 10 \%$ dextrose is given over 1 minute, immediately followed by a continuous glucose infusion of 5 to $8 \mathrm{mg} / \mathrm{kg}$ per minute is standard. This minibolus infusion results in rapid correction of blood glucose level (usually within 1 minute), and relative stability of values between approximately 70 and $80 \mathrm{mg} / \mathrm{dL}$ occurs after that with the continuous infusion of 5 to $8 \mathrm{mg} / \mathrm{kg}$ per minute. This infusion rate is based on the rate of endogenous glucose production in healthy newborn infants.

Careful assessment of the initial clinical response after the minibolus infusion is essential, especially if the indication for the infusion was a seizure because of variability in response to blood glucose level; a second minibolus infusion may be necessary.

Continued careful monitoring of clinical response and blood glucose level also is important because certain infants (e.g., those with hyperinsulinism) may require higher maintenance doses of glucose, whereas some infants require lower maintenance doses to avoid hyperglycemia. In general, after blood glucose levels are stable at 70 to $100 \mathrm{mg} / \mathrm{dL}$, the dextrose concentration in the infusion may be decreased by 1 to $2 \mathrm{mg} / \mathrm{kg}$ per minute every 6 to 12 hours. Glucose levels are monitored closely and should be maintained at more than $50 \mathrm{mg} / \mathrm{dL}$. It is essential not only to correct but also to avoid hyperglycemia. For infants whose glucose levels do not increase adequately despite higher infusion rates of at least 10 to $12 \mathrm{mg} / \mathrm{kg}$ per minute or who require infusion rates of more than $12 \mathrm{mg} / \mathrm{kg}$ per minute or if hypoglycemia recurs, hyperinsulinism should be considered. Treatment for the latter condition includes diazoxide or octreotide (both suppress insulin secretion) or pancreatic surgery. Less likely causes include specific hormonal defects (e.g., hypopituitarism) or a metabolic disorder that requires specific therapy. Hydrocortisone, previously used in this context 
at $5 \mathrm{mg} / \mathrm{kg}$ every 12 hours, has increased gluconeogenesis (from protein sources) and decreased peripheral glucose utilization. This agent has been used less in recent years; if administered, it should be discontinued as soon as feasible.

Feeding of the hypoglycemic baby has several layers to unfold. A baby we expect to feed well, such as the term healthy infant, but is feeding poorly, should be evaluated as to the cause. It is inappropriate to gavage this type of baby without understanding the reason for the poor feeding. Since hypoglycemia causes poor feeding, this is another reason for the inability of the baby to feed. In circumstances where an evaluation is being performed to determine if a congenital hypoglycemic disorder is present, some investigators suggest that the feeding should be given only as a constant-rate nasogastric drip to avoid hormonal excursion and simplify the assessment.

\section{"Some have advocated using dextrose gel to stabilize infants with very low glucose while preparing for a peripheral intravenous line. This treatment approach has not yet been widely adopted in the United States."}

In infants with hypoglycemia being breastfed, establishing adequate milk let down, and ingestion may take 1-2 days. In these cases, the introduction of formula may create some controversy with respect to the success in subsequently establishing breastfeeding. If causes for the hypoglycemia have been assessed and one is convinced the mildly low glucose level is secondary to transitional neonatal hypoglycemia, some centers used oral dextrose gel as a supplemental management option. This gel can be applied directly to mucosal surfaces of the mouth, including buccal and lingual surfaces, with rapid access to the circulation. The estimated rise in blood glucose concentration following dextrose gel is $0.4 \mathrm{mmol} / \mathrm{L}$ ( $40 \mathrm{~g}$ of dextrose per $100 \mathrm{~mL}$, administered at 200 $400 \mathrm{mg} / \mathrm{kg}$ ). The risk of rebound hypoglycemia, however, is a possibility and needs close monitoring. Some have advocated using dextrose gel to stabilize infants with very low glucose while preparing for a peripheral intravenous line. This treatment approach has not yet been widely adopted in the United States.

\section{Symptoms:}

Neonates with hypoglycemia may be asymptomatic or manifest nonspecific symptoms similar to many disorders in newborn infants. The symptoms of hypoglycemia reflect brain responses to glucose deprivation and consist of 2 types of reactions: neurogenic and neuroglycopenic. The neurogenic symptoms are caused by activation of sympathetic discharge and include tachycardia, tremors, lethargy and diaphoresis. The neuroglycopenic symptoms include desaturations, apnea, bradycardia, respiratory distress, hypothermia, seizures, and coma. Limited data suggest that the glucose thresholds for both neurogenic and neuroglycopenic responses to hypoglycemia may not be different in newborn infants than in older children and adults.

Physiologically, hypoglycemic symptoms may present when glucose delivery is inadequate to meet glucose demand and can occur over a range of glucose concentrations, depending on the status of the infant. For example, a 2-hour-old healthy infant who has a blood glucose of $30 \mathrm{mg} / \mathrm{dL}$ ( $1.7 \mathrm{mmol} / \mathrm{L}$ ) might not demonstrate impaired organ function, but a stressed infant might demonstrate physiologic hypoglycemia at a blood glucose concentration of 50 $\mathrm{mg} / \mathrm{dL}(2.8 \mathrm{mmol} / \mathrm{L}$ ) if the rate of glucose delivery to the brain is less than the rate of glucose utilization. Given the neonates' proportionately greater demand for glucose by the brain, many advise that there is no reason to assume that a lower threshold for intervention is likely appropriate in neonates. Even if the health care team is astute at detecting hypoglycemia-related symptoms that usually are caused by either counter-regulation or the absence of alternate substrates to fuel brain oxidative metabolism, the cause for lack of symptoms remains evasive. The asymptomatic state may relate to a lack of the compensatory counter-regulatory hormonal response similar to the unawareness state described in older children, which may be ominous. The greatest variance in the management of hypoglycemia is seen in the asymptomatic infant diagnosed soon after birth, during the first 2 hours. Controlled studies examining the benefits or impact of this early feeding on recovery from hypoglycemia have not been performed. It has been suggested that as many as $10 \%$ of healthy infants who are the appropriate size for gestational age develop transient asymptomatic hypoglycemia, which in most cases is managed by the initiation of early, normal feeding. Considering that data show that plasma glucose concentrations in transitional hypoglycemia are at their lowest in the first 2 hours and are remarkably stable and relatively unaffected by the timing of initial feeding or interval between feedings and that the lowest level of glucose concentration, the hope that the feeding will resolve the low glucose level seems at some level overly hopeful.

\section{"The combination of hypoglycemia, hypoxemia, and/or ischemia leads to greater cerebral metabolic derangement than observed with either condition alone. The deleterious effects of hypoxemia secondary to fetal distress are potentiated after delivery if low plasma glucose concentrations occur."}

\section{Hypoglycemia and Asphyxia}

Pathogenetic factors in asphyxia resulting in hypoglycemia are (1) enhanced cerebral utilization of glucose secondary to anaerobic glycolysis, (2) glycogen depletion secondary to intrapartum stressinduced catecholamine release, and (3) hypersecretion of insulin. Hypoglycemia secondary to asphyxia usually has its onset on the first day of life (although usually later than in the early transitional group), duration is variable, the degree is relatively mild, and response to glucose therapy is prompt. However, in the severely asphyxiated infant, the onset is earlier, the degree is marked, and depending on the degree of hyperinsulinism, the response to glucose may not be prompt. Affected infants often require high glucose infusion rates to maintain a normal glucose concentration, suggesting either an increased insulin concentration or increased insulin sensitivity. Because asphyxia also increases plasma glucagon, interleukin-6, hydrocortisone, and other counter-regulatory 
hormones, with associated changes in insulin receptor binding, it can also induce an insulin-resistant state.

The combination of hypoglycemia, hypoxemia, and/or ischemia leads to greater cerebral metabolic derangement than observed with either condition alone. The deleterious effects of hypoxemia secondary to fetal distress are potentiated after delivery if low plasma glucose concentrations occur. Depending on the severity and duration, either alone would not necessarily cause brain injury but may do so when acting in concert. An ischemic insult is significant because of the likelihood of ischemia to certain brain regions during hypoglycemia with moderate hypotension. This likelihood relates to the impairment of vascular autoregulation. Intervening with blood pressure and glucose control is critically important. Similar to pure hypoxemia, the degrees of ischemia and hypoglycemia alone would not cause brain injury might do so when acting together.

\section{"The dominant finding has been abnormal signal intensity by magnetic resonance imaging (MRI) in the parietal-occipital region. The involved areas exhibit restricted diffusion on diffusion-weighted MRI (DWI). The topography is seen better in the acute stage by DWI than by conventional MRI."}

\section{Technical issues:}

There are many potential artifacts in measuring plasma glucose concentrations:

1. Venous and capillary blood have lower glucose concentrations than the concentration of arterial plasma glucose to which the brain is exposed.

2. The glucose concentration in plasma is approximately $15 \%$ higher than in whole blood due to the volume occupied by red blood cells,

3. Glucose concentrations in whole blood specimens decline rapidly with delays in processing due to consumption by red and white blood cells

4. Glucose measurements by bedside glucometer testing have a $15 \%$ imprecision inherently and are also highly susceptible to multiple artifacts of sampling and operator errors.

\section{Imaging:}

The dominant finding has been abnormal signal intensity by magnetic resonance imaging (MRI) in the parietal-occipital region. The involved areas exhibit restricted diffusion on diffusion-weighted MRI (DWI). The topography is seen better in the acute stage by DWI than by conventional MRI. Magnetic resonance spectroscopy shows no or mild lactate elevations with advanced lesions, indicating that the lesion is not basically ischemic but rather related to glucose deprivation. Although $10 \%$ to $15 \%$ of the lesions resolve, most are followed in subsequent weeks and months by loss of cerebral cortex and white matter, often with ventricular dilation. A more diffuse pattern of cerebral cortical injury may occur with very severe hypoglycemia. In general, the posterior cerebral involvement includes both cortex and underlying white matter, seen best acutely by DWI imaging. Conventional MRI sequences often show predominantly white matter involvement.

\section{Outcome:}

Mortality and neurological outcome in neonatal hypoglycemia relate to the level and duration of the low plasma glucose and the timeliness of appropriate intervention. The most common sequelae include developmental delay, mental retardation, visual disturbances, and seizure disorders.

\section{Summary of Case:}

This case involved an AGA term baby who was a healthy, normally grown, and normally responsive fetus entering at the onset of labor. Over time and prolonged labor with excessive uterine activity, his heart rate pattern worsened, especially during the $2^{\text {nd }}$ stage of labor resulting in an emergency Cesarean section with vacuum assistance needed to deliver the fetal head. He needed some blow-by oxygen and had Apgars of $6^{1}$ and $8^{5}$; the arterial cord $\mathrm{pH}$ was 7.09. He had a rare cry, and at 20 minutes, he was tachycardic and tachypneic with intercostal retractions. Nevertheless, he was sent to room-in with his mother. His tachypnea and tachycardia, likely secondary from his fetal distress and potentially traumatic delivery were attempts to increase cardiac output and blow off $\mathrm{CO}_{2}$. Any baby with either this history or these symptoms should have been admitted directly to the NICU from the delivery room; a blood gas and a chest radiograph should have been done; and interventions provided to stabilize his cardiovascular, respiratory, and metabolic conditions. He would have had a peripheral intravenous line with glucose, and his plasma glucose levels would have been monitored intermittently. However, because he was sent to room-in with his mother and failed to show interest in feeding, he was brought to the normal nursery where he continued to have an elevated RR and HR, became lethargic, and developed multiple desaturations. Eventually, markedly low bedside glucose prompted gavage feedings, an inappropriate approach to bypass the respiratory distress. The baby eventually was admitted to the NICU, where a bolus of glucose was administered $31 / 2 \mathrm{~h}$ after birth, $2 \mathrm{~h}$ after the first of several unreadable glucose evaluations. The baby was minimally responsive in the NICU for the first 24 hours, developed seizures the following day, and had a stormy course, including DIC. MRI showed intraventricular, parenchymal, and subdural hemorrhages. On follow-up, he had severe developmental delays, cerebral palsy, and a seizure disorder.

\section{"Preventive care was required during labor and in the neonatal period. Avoiding fetal distress (avoiding the need to rescue the fetus), admitting the newborn directly to the NICU would most probably have resulted in a normal child today."}

Preventive care was required during labor and in the neonatal period. Avoiding fetal distress (avoiding the need to rescue the 
fetus), admitting the newborn directly to the NICU would most probably have resulted in a normal child today.

\section{Conclusion:}

Babies with symptoms should not room in with their mothers or go to the normal nursery. Even asymptomatic babies born after fetal distress need to have their glucose monitored. Prevention of hypoglycemia is essential. Screening protocols should be expanded to include a larger target population and include babies born a) after fetal distress, cesarean section for intolerance to labor and complicated deliveries, with cord acidemia b) to mothers with hypertension; c) to mothers who received glucose infusions, especially boluses during fetal decompensation intervals; d) with IUGR e). with postdates f) with higher length compared to weight (low Ponderal Indices), g) born to mothers who received sympathomimetics (terbutaline, ritodrine,) and propranolol. Prompt intervention with intravenous glucose is critically important for the hypoglycemic newborn.

\section{Suggested Reading:}

1. Committee on F, Newborn, Adamkin DH. Postnatal glucose homeostasis in late-preterm and term infants. Pediatrics. 2011;127(3):575-9. Epub 2011/03/02. doi: 10.1542/ peds.2010-3851. PubMed PMID: 21357346.

2. ElHassan NO, Schaefer EW, Gonzalez B, Nienaber T, Brion LP, Kaiser JR. Early Transient Hypoglycemia and Test Performance in At-Risk Newborns. Am J Perinatol. 2021. Epub 2021/08/01. doi: 10.1055/s-0041-1732380. PubMed PMID: 34331300.

3. Harding JE, Harris DL, Hegarty JE, Alsweiler JM, McKinlay CJ. An emerging evidence base for the management of neonatal hypoglycaemia. Early Hum Dev. 2017;104:51-6. Epub 2016/12/19. doi: 10.1016/j.earlhumdev.2016.12.009. PubMed PMID: 27989586; PubMed Central PMCID: PMCPMC5280577.

4. Harris DL, Weston PJ, Gamble GD, Harding JE. Glucose Profiles in Healthy Term Infants in the First 5 Days: The Glucose in Well Babies (GLOW) Study. J Pediatr. 2020;223:3441 e4. Epub 2020/05/10. doi: 10.1016/j.jpeds.2020.02.079. PubMed PMID: 32381469.

5. Harris DL, Weston PJ, Harding JE. Alternative Cerebral Fuels in the First Five Days in Healthy Term Infants: The Glucose in Well Babies (GLOW) Study. J Pediatr. 2021;231:816 e2. Epub 2020/12/30. doi: 10.1016/j.jpeds.2020.12.063. PubMed PMID: 33373670.

6. Hawdon JM. Definition of neonatal hypoglycaemia: time for a rethink? Arch Dis Child Fetal Neonatal Ed. 2013;98(5):F3823. Epub 2013/05/07. doi: 10.1136/archdischild-2012-303422. PubMed PMID: 23645576.

7. Salhab WA, Wyckoff MH, Laptook AR, Perlman JM. Initial hypoglycemia and neonatal brain injury in term infants with severe fetal acidemia. Pediatrics. 2004;114(2):361-6. Epub 2004/08/03. doi: 10.1542/peds.114.2.361. PubMed PMID: 15286217.

8. Stanley CA, Rozance PJ, Thornton PS, De Leon DD, Harris $D$, Haymond MW, et al. Re-evaluating "transitional neonatal hypoglycemia": mechanism and implications for management. J Pediatr. 2015;166(6):1520-5 e1. Epub 2015/03/31. doi: 10.1016/j.jpeds. 2015.02.045. PubMed PMID: 25819173; PubMed Central PMCID: PMCPMC4659381.

9. Tam EW, Haeusslein LA, Bonifacio SL, Glass HC, Rogers $E E$, Jeremy RJ, et al. hypoglycemia is associated with increased risk for brain injury and adverse neurodevelopmental outcome in neonates at risk for encephalopathy. $\mathrm{J} \mathrm{Pe}$ diatr. 2012;161(1):88-93. Epub 2012/02/07. doi: 10.1016/j. jpeds.2011.12.047. PubMed PMID: 22306045; PubMed Central PMCID: PMCPMC3346850.
10. Thornton PS, Stanley CA, De Leon DD, Harris D, Haymond MW, Hussain K, et al. Recommendations from the Pediatric Endocrine Society for Evaluation and Management of Persistent Hypoglycemia in Neonates, Infants, and Children. J Pediatr. 2015;167(2):238-45. Epub 2015/05/11. doi: 10.1016/j.jpeds.2015.03.057. PubMed PMID: 25957977.

11. Wong DS, Poskitt KJ, Chau V, Miller SP, Roland E, Hill A, et al. Brain injury patterns in hypoglycemia in neonatal encephalopathy. AJNR Am J Neuroradiol. 2013;34(7):1456-61. Epub 2013/02/26. doi: 10.3174/ajnr.A3423. PubMed PMID: 23436054 ; PubMed Central PMCID: PMCPMC8051482.

Disclosures: The authors have indicated no conflicts of interest.

NT
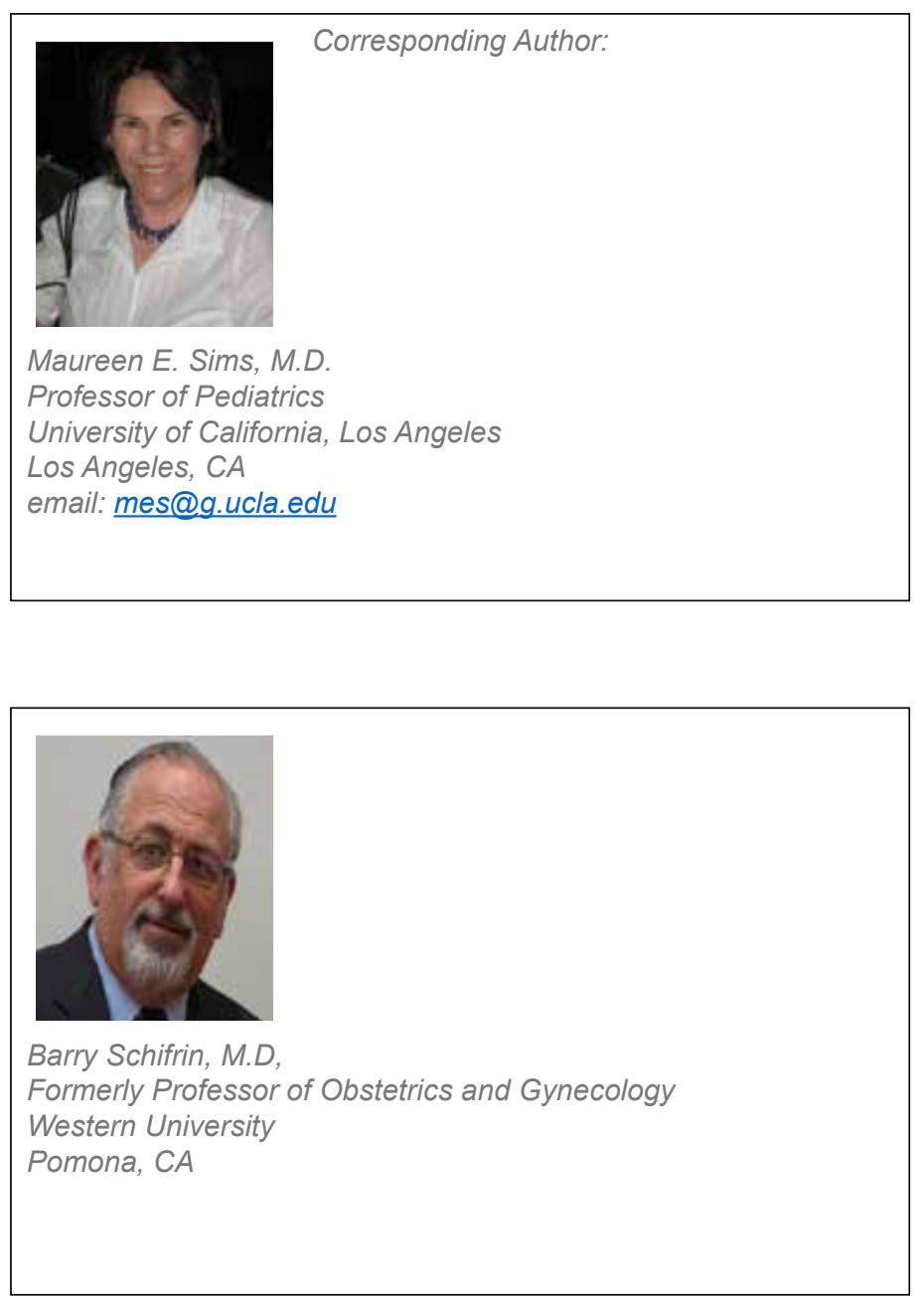
Readers can also follow NEONATOLOGY TODAY via our Twitter Feed @NEOTODAY 